\title{
RIQUEZA DE LA FAMILIA ORCHIDACEAE EN LA ZONA DE VISITANTES DEL PARQUE NACIONAL CERRO AZUL MEÁMBAR DE HONDURAS
}

\author{
KLAus W. Wiese \\ Museo de Historia Natural, UNAH, Ciudad Universitaria, Tegucigalpa, Honduras \\ klaus.wiese@unah.edu.hn
}

\begin{abstract}
Resumen. Se presentan las especies de orquídeas encontradas en la zona de visitas del Parque Nacional Cerro Azul Meámbar (PanACAM), Comayagua, Honduras. A partir de las observaciones de orquídeas se generó una matriz de presencia-ausencia mensual basada en la floración observada de cada especie. En esta matriz se hizo un análisis de riqueza de especies usando el estimador jackknife de primer orden. Se agrupan los meses del año en función de la riqueza de las especies de orquídeas con flor para cada mes. Se registran 101 especies más de orquídeas para el parque y se predice la presencia de aproximadamente 140 en su zona de visitantes.
\end{abstract}

Abstract. A list of orchids found in the visitors zone of Parque Nacional Cerro Azul Meámbar, Comayagua, Honduras, is presented. A monthly absence- presence matrix was made for the orchids found with flowers of each species; with this matrix we made a richness analysis using the first order jackknife estimator. The months of the year were grouped in function of the orchid species in flower for each month. An additional 101 species of orchids are reported for the park, and 140 species are predicted for the visitor's zone.

Palabras Clave / Key Words: Bosque Muy Húmedo Subtropical, Subtropical Wet Forest, Honduras, Orchidaceae, Panacam, Proyecto Aldea Global

Introducción. Honduras tiene registradas 7,299 plantas vasculares espermatófitas; de éstas, las familias más numerosas son las leguminosas y las orquídeas (Orchidaceae 693 especies, 1 subespecie y 8 variedades) (Nelson, 2008, 2010, Archila 2013, Vega et al. 2014).

La familia Orchidaceae, además de ser una de la familias con flor más numerosas en Honduras, es una de las más numerosas en el planeta tierra (Dressler 2005). Las orquídeas despiertan gran interés en el mundo científico, turístico, industrial (comercial-ornamental) y conservación (Hágsater \& Dumont 1996), siendo este interés una de las principales razones para abordar este grupo de plantas.

Existen pocos estudios sobre la flora del Parque Nacional Cerro Azul Meámbar (Panacam). Hazlett (1980) publicó sobre la vegetación del parque haciendo énfasis en árboles, pero no menciona las especies de orquídeas observadas. Hawkins (1993) reporta tres especies de orquídeas, Maxillaria cucullata, Arpophyllum giganteum y una especie desconocida. En un anexo de esa publicación se presenta la lista de plantas depositadas en el Herbario de la Escuela Nacional de Ciencias Forestales (ESNACIFOR) que provienen de Panacam, sin definir la localidad específica de cada espécimen, listando las especies de orquídeas Encyclia (= Prosthechea) brassavolae, Lockhartia hercodonta y Pleurothallis cardiothallis, todas colectadas por Donald Hazlett.

Borjas et al. (1997) reportan las orquídeas Maxillaria cucullata y Habenaria sp., en los planes de manejo para PanaCam de 1998, 2000 y, el más reciente para el período 2012 - 2016 (AFE-COHDEFor, 1998, 2000, ICF 2012), se menciona la presencia de Epidendrum comayaguense (= Epidendrum cerinum) y de varias otras especies numeradas como desconocidas. Vega et al. (2014) reportan Specklinia spectabilis y Microchilus killipii para la zona de visitantes de Panacam.

Nelson y Ortiz (2007) publican la colección de orquídeas del Herbario TEFH en donde se menciona Epidendrum radicans para la localidad Cerro Azul Meámbar, así como Encyclia alata y Oncidium graminifolium para la localidad de Meámbar, Comayagua. Para río Yure se enumeran las especies Dimerandra emarginata, Encyclia nematocaulon, Epidendrum schlechterianum, Liparis nervosa, 
Notylia barkeri, Polystachya foliosa (= Polystachya caracasana, siguiendo a Peraza-Flores et al. 2011), Prosthechea radiata, Barkeria obovata, Catasetum integerrimum y Cattleya (= Guarianthe) skinneri. Finalmente se menciona la localidad Santa Elena, Comayagua, con la especie Sobralia decora.

También se revisaron algunas bases de datos digitales de herbarios. En Tropicos (2015) de los 1206 registros de orquídeas para Honduras disponibles, se encontraron 15 registros de orquídeas para PANACAM; de éstas, 12 son colectas de James B. Edwards realizadas entre 1932 y 1933: Brassia caudata, Comparettia falcata, Cryptarrhena lunata, Dichaea acroblephara, Habenaria lactiflora, H. repens, Hexisea (= Scaphyglottis) imbricata, Jacquiniella cobanensis, Oncidium ensatum, Oncidium leucochilum, Psygmorchis (= Erycina) cristagalli y Trichocentrum lindenii. Las tres plantas restantes fueron colectadas por Randall J. Evans; William E. Harmon \& José A. Fuentes y Thomas Hawkins, Bruce Allen \& Randall J. Evans, siendo estas Epidendrum verrucosum, Epidendrum difforme (probablemente una especie afín, ya que esta especie es endemica de las Antillas Menores) y Maxillaria cucullata. Ninguna de éstas plantas se encuentra en las zona de visitantes del Parque. En el herbario virtual C. V. Starr del Jardín Botánico de New York existe registro de 56 orquídeas de Honduras (C. V. Starr Virtual Herbarium, 2015), ninguna de éstas dentro de los límites del PanACAM. En los Especímenes Neotropicales del Museo Field de Chicago se encontraron 42 registros de orquídeas para Honduras, de éstas, ninguna corresponde al área del PanACAm.

Este trabajo tiene por objetivo presentar las especies de orquídeas de la zona de visitantes del Parque Nacional Cerro Azul Meámbar como una herramienta para entender las orquídeas en ese sitio. Es un insumo para elaborar estrategias eficientes para su manejo y conservación.

Área de estudio. El Panacam está ubicado en los departamentos de Comayagua y Cortés, Honduras. Esta comprendido entre los 600 y $2047 \mathrm{msnm}$, fue creado en el año 1987 bajo la ley de bosques nublados 87-87 (Cruz 1993). Es comanejado por el Proyecto Aldea Global (PAG). Su zona de visitantes está definida en este estudio empezando con la calle que une Santa Elena con el Centro de Visitantes El Pino de Panacam $(4.1 \mathrm{~km})$, los senderos Sinaí $(5.2 \mathrm{~km})$ y Los Vencejos $(0.6 \mathrm{~km})$ dentro del área de uso público del Parque (Fig. 1). En términos de área, el recorrido general representa aproximadamente 4.04 hectáreas (0.0404 km cuadrados).

La zona de visitantes es un Bosque Muy Húmedo Sub Tropical (Holdridge 1962), y se encuentra entre los 600 y 1200 msnm. Se puede separar en dos tipos según su intervención actual. Primero, el camino entre Santa Elena y El Pino está muy intervenido con agricultura tradicional: café, asentamientos humanos, proyecto de generación eléctrica (represa Yure). Segundo, dentro del área de uso público se presenta un paisaje no intervenido de bosque latifoliado.

La precipitación promedio anual es de 2,892.3 $\mathrm{mm}$, el mes de septiembre presenta el promedio más alto con $459.4 \mathrm{~mm}$ y el mes de marzo tiene promedio más bajo con $66.4 \mathrm{~mm}$. La temperatura promedio anual es de $21.44^{\circ} \mathrm{C}$, el mes más frío es diciembre con $17.86^{\circ} \mathrm{C}$ y el más caliente es mayo con $23.53^{\circ} \mathrm{C}$. Estos datos fueron extraídos de la estación Santa Elena de la Empresa Nacional de Energía Eléctrica (ENEE).

\section{Metodología}

Esfuerzo -. Se realizaron 18 visitas que comenzaron en enero de 2011 y finalizaron en marzo de 2014. En 2011, se visitó en los meses de enero, marzo, mayo, agosto, octubre y diciembre. En 2012, febrero, abril, junio, julio y septiembre. En 2013, enero, abril, julio, septiembre, noviembre y diciembre, y durante 2014 se hizo una visita en marzo. Se procuró visitar el área de interés al menos una vez por cada mes del año buscando tener acceso a la mayor representatividad temporal de orquídeas en flor. En cada viaje se recorría la zona de interés haciendo el mismo esfuerzo: sendero Sinaí ocho horas, Sendero Los Vencejos, dos horas, camino entre el Centro de Visitantes Pino y Santa Elena, tres horas. Cada orquídea encontrada con flor era fotografiada con detalle y se tomaban las medidas que permitieran su determinación. No se colectó ninguna muestra pues no se contó con los permisos de colecta correspondientes; las imágenes de cada especie están consolidadas en una base de datos a la que se puede acceder contactando al autor.

Riqueza y agrupación -. Para estimar la riqueza esperada de especies, se usó una matriz de presencia- 


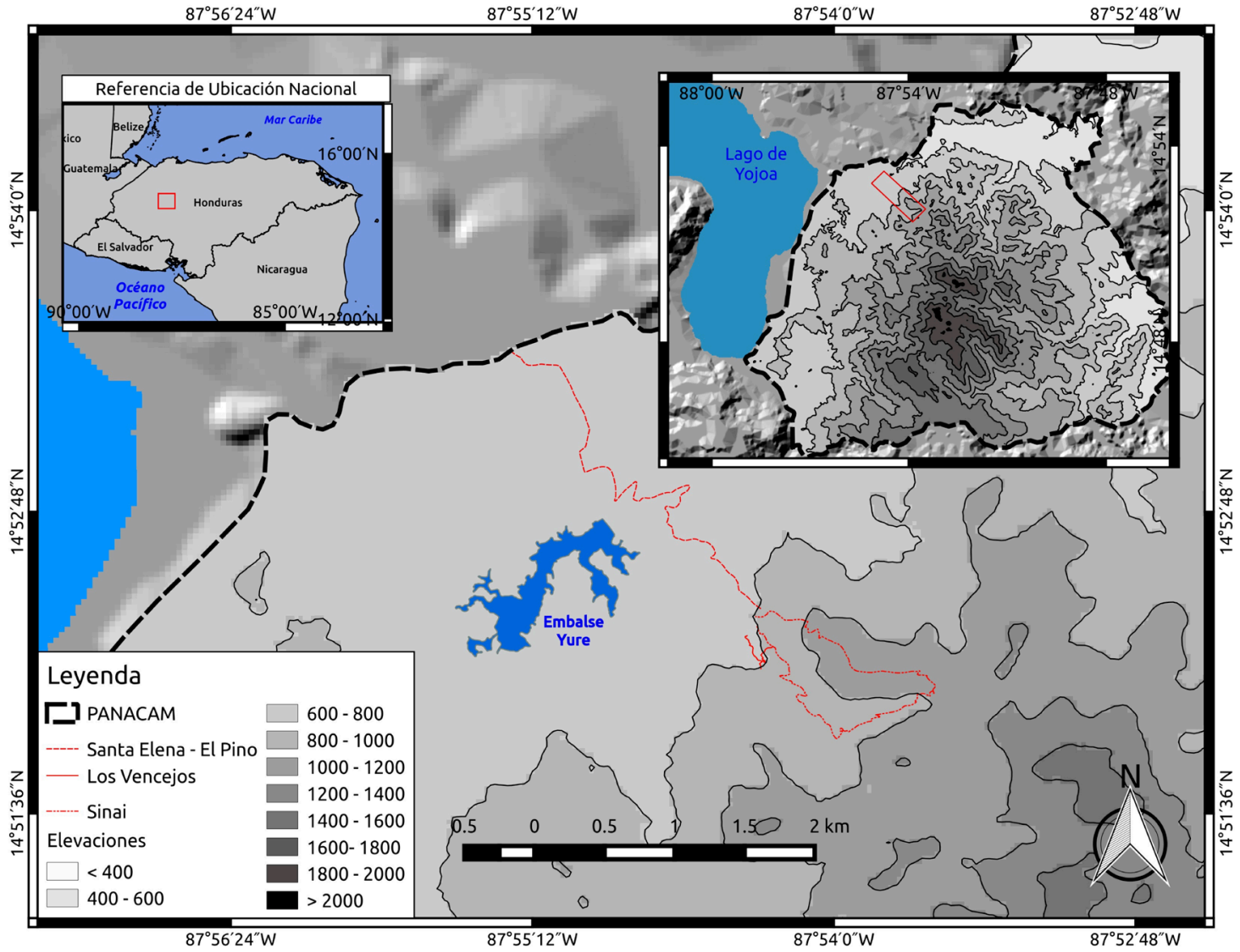

FIgURA 1. Zona de visitantes Parque Nacional Cerro Azul Meámbar (Elaborado con QGIS Development Team [2015]).

ausencia de orquídeas en flor por cada mes. Una orquídea con flor se contabilizaba como presencia para la especie que representaba. Esta matriz fue analizada usando el paquete fossil (Vavrek 2011) del programa R (R Core Team 2013) con el algoritmo jackknife de primer orden.

Los meses fueron agrupados según su riqueza, usando una matriz de distancia euclidiana entre los meses para luego ser graficados en un árbol binario unrooted usando el paquete APE de la aplicación R (Paradis et al. 2004).

\section{Resultados}

Listado de especies -. Adicionalmente a las especies que se lograron encontrar en flor, se agregaron orquídeas que se podían determinar, hasta donde fuese posible, en estado estéril. En la Tabla 1 se presenta la lista de orquídeas encontradas en la zona de visitantes de PANACAM ordenadas alfabéticamente. Los nombres usados para las especies son en su mayoría los aceptados por Govaerts et al. (2011) y Chase et al. (2015). Además, se incluye si la especie es cultivada, invasora y comentarios.

Análisis de riqueza -. El resultado de la acumulación de especies (Fig. 2) utilizando jackknife de primer orden (Vavrek 2011) proyecta la presencia de aproximadamente 140 especies en las zona de visitantes de PANACAM.

Agrupación de meses según riqueza en floración -. Al agrupar los meses en función de la riqueza de las especies en floración encontramos dos grupos: G-1 y G-2 (ver Fig. 3). El primero presenta los picos más altos de floración. Este grupo se divide en dos subgrupos G-1a y G-1b. G-1a está representado por los meses de agosto, septiembre, octubre y noviembre; G-1b se forma en los meses diciembre, enero y febrero. Se hace esta segregación pues a pesar que en estos meses se 
Tabla 1. Orquídeas de la zona de visitantes del Panacam.

\begin{tabular}{|c|c|}
\hline Arpophyllum sp. & Gongora truncata Lindl.* \\
\hline Arundina graminifolia (D.Don) Hochr. ${ }^{*} 1$ & Guarianthe skinneri (Bateman) Dressler \& W.E.Higgins ${ }^{1}$ \\
\hline Bletia purpurea (Lam.) A.DC * & Habenaria sp. \\
\hline Brassavola sp.* & lonopsis utricularioides (Sw.) Lindl.* \\
\hline Brassia verrucosa Bateman ex Lindl.* & Isochilus linearis (Jacq.) R.Br. Schltr. ${ }^{*}$ \\
\hline Campylocentrum schiedei (Rchb.f.) Benth. ex Hemsl. * & Jacquiniella teretifolia (Sw.) Britton \& P.Wilson* \\
\hline Catasetum integerrimum Hook. & Lepanthes acuminata Schltr.* \\
\hline Comparettia falcata Poepp. \& Endl. ${ }^{*}$ & scopula Schltr.* \\
\hline Cranichis wageneri Rchb.f.* & sp. $1^{*}$ \\
\hline Cranichis sp. * & sp. $2^{*}$ \\
\hline Cyclopogon comosus (Rchb.f.) Burns-Bal. \& E.W.Greenw. ${ }^{*}$ & Lepanthopsis floripecten (Rchb.f.) Ames* \\
\hline cranichoides (Griseb.) Schltr.* & Lockhartia hercodonta Rchb.f. ex Kraenzl. \\
\hline Cyrtopodium macrobulbon (Lex.) G.A.Romero \& Carnevali * 3 & Malaxis sp. * \\
\hline Dichaea glauca (Sw.) Lindl.* & Maxillaria aciantha Rchb.f. ${ }^{*}$ \\
\hline hystricina Rchb.f.* & anceps Ames \& C.Schweinf. ${ }^{*}$ \\
\hline muricatoides Hamer \& Garay* & cobanensis Schltr.* \\
\hline panamensis Lindl. ${ }^{*}$ & friedrichsthalii Rchb.f.* \\
\hline trulla Rchb.f.* & fulgens (Rchb.f.) L.O.Williams * \\
\hline tuerckheimii Schltr.* & ringens Rchb.f.* \\
\hline sp. ${ }^{*}$ & uncata Lindl. ${ }^{*}$ \\
\hline Dimerandra emarginata (G.Mey.) Hoehne & variabilis Bateman ex Lindl.* \\
\hline Dinema polybulbon (Sw.) Lindl.* & Mormodes ephippilabia Fowlie* \\
\hline Elleanthus capitatus (Poepp. \& Endl.) Rchb.f. ${ }^{*}$ & sotoana Salazar* \\
\hline caricoides Nash* & Nidema boothii (Lindl.) Schltr.* \\
\hline graminifolius (Barb.Rodr.) Løjtnant* & Notylia barkeri Lindl. \\
\hline poiformis Schltr.* & Oeceoclades maculata (Lindl.) Lindl.. ${ }^{2}$ \\
\hline Encyclia tuerckheimii Schltr.* & Oncidium sphacelatum Lindl.. 1 \\
\hline sp. ${ }^{*}$ & Ornithocephalus numenius Toscano \& Dressler * \\
\hline Epidendrum flexuosum G.Mey. ${ }^{*}$ & Pelexia funckiana (A.Rich. \& Galeotti) Schltr. * \\
\hline glumarum Hamer \& Garay* & Platystele oxyglossa (Schltr.) Garay* \\
\hline isomerum Schltr.* & stenostachya (Rchb.f.) Garay* \\
\hline melistagum Hágsater* & sp.* \\
\hline mixtum Schltr.* & Platythelys querceticola (Lindl.) Garay* 4 \\
\hline nocturnum Jacq. ${ }^{*}$ & Pleurothallis cardiothallis Rchb.f. \\
\hline paranthicum Rchb.f.* & correllii Luer* \\
\hline radicans Pav. ex Lindl. & pansamalae Schltr.* \\
\hline rigidum jacq.* & pruinosa Lindl.* \\
\hline veroscriptum Hágsater* & sp. ${ }^{*}$ \\
\hline yojoaënse Hágsater \& L.Sánchez* & Polystachya caracasana Rchb.f. \\
\hline Erycina crista-galli (Rchb.f.) N.H.Williams \& M.W.Chase & Prescottia stachyodes (Sw.) Lindl.* \\
\hline Gongora leucochila Lem.* & Prosthechea baculus (Rchb.f.) W.E.Higgins* \\
\hline
\end{tabular}


TABLA 1. Sigue.

\begin{tabular}{|c|c|}
\hline Prosthechea cochleata (L.) W.E.Higgins * 2 & Specklinia spectabilis (Ames \& C.Schweinf.) Pupulin \& Karremans \\
\hline livida (Lindl.) W.E.Higgins * & tribuloides (Sw.) Pridgeon \& M.W.Chase * \\
\hline pygmaea (Hook.) W.E.Higgins * & Stanhopea oculata (Lodd.) Lindl.* \\
\hline radiata (Lindl.) W.E.Higgins & sp. * \\
\hline Psilochilus macrophyllus (Lindl.) Ames * & Stelis gracilis Ames* \\
\hline Restrepia muscifera (Lindl.) Rchb.f. ex Lindl. * & megachlamys (Schltr.) Pupulin * \\
\hline Sacoila lanceolata (Aubl.) Garay * & segoviensis (Rchb.f.) Pridgeon \& M.W.Chase * \\
\hline Scaphyglottis confusa (Schltr.) Ames \& Correll * & sp. $1^{*}$ \\
\hline lindeniana (A.Rich. \& Galeotti) L.O.Williams * & sp. 2 * \\
\hline livida (Lindl.) Schltr. * & $\mathrm{sp} .3^{*}$ \\
\hline longicaulis S.Watson* & Trichocentrum ascendens (Lindl.) M.W.Chase \& N.H.Williams * \\
\hline prolifera (R.Br.) Cogn. * & luridum (Lindl.) M.W.Chase \& N.H.Williams * \\
\hline Sobralia decora Bateman & Trichopilia tortilis Lindl. * \\
\hline macrantha Lindl. * & Trichosalpinx blaisdellii (S.Watson) Luer * \\
\hline Specklinia glandulosa (Ames) Pridgeon \& M.W.Chase * & Trigonidium egertonianum Bateman ex Lindl. * 5 \\
\hline grobyi (Bateman ex Lindl.) F.Barros * & \\
\hline
\end{tabular}

${ }^{*}=$ Registrada en el presente trabajo, 1 = Cultivada, 2 = Invasora, 3 = Comúnmente confundida en Honduras con C. punctatum (Romero-González et al. 2008), 4 = Género trasladado a Aspidogyne por Chase et al. (2015), 5 = Género trasladado a Maxillaria por Chase et al. (2015).

presenta la mayor cantidad de orquídeas en flor se observa una cantidad mayor en la agrupación G-1b y se considera importante mencionar que existen diferencias entre los meses que conforman el grupo G-1. G-2 está conformado por marzo, abril, mayo, junio y julio.

Discusión. Hasta el momento se había reportado la cantidad de 38 especies de orquídeas para el área total de Panacam (Hawkins 1993, Borjas et al. 1997, AfECohDEFor 1998, 2000, Nelson \& Ortiz 2007, ICF 2012, Vega et al., 2014, Tropicos 2014, C.V. Starr Virtual Herbarium, 2015). En el presente estudio se

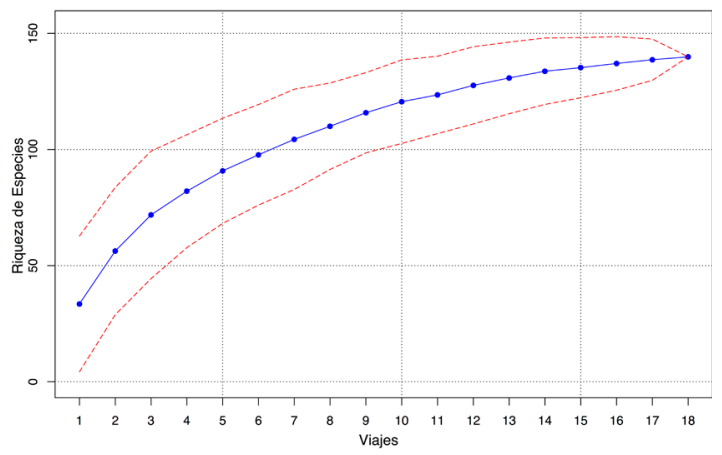

Figura 2. Curva de acumulación de especies. encontraron 116 especies que pertenecen a 54 géneros, 17 subtribus, nueve tribus y dos subfamilias, usando la clasificación propuesta por Chase et al. (2015), de las cuales 101 son nuevos registros para el Parque. Se pueden enumerar por lo menos 139 especies de orquídeas para el Parque.

Se predice la presencia de aproximadamente 140 especies de orquídeas en la zona de visitantes de

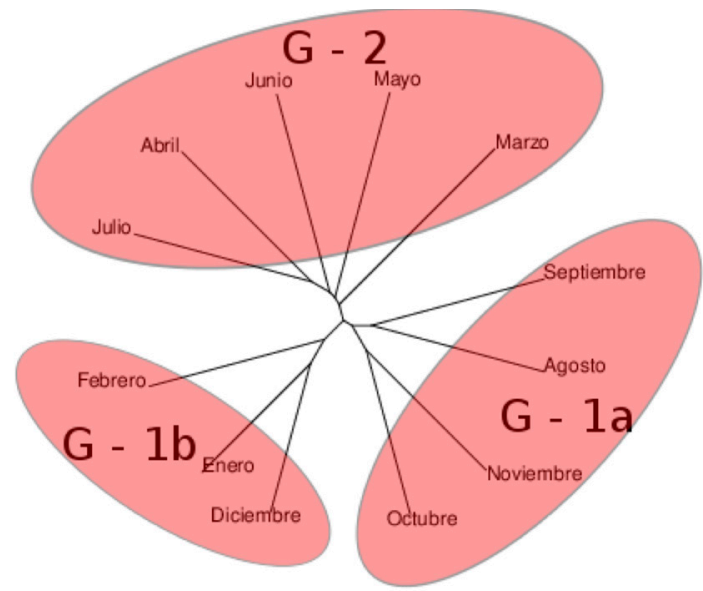

Figura 3. Agrupación de meses por riqueza (unrooted binary tree). 


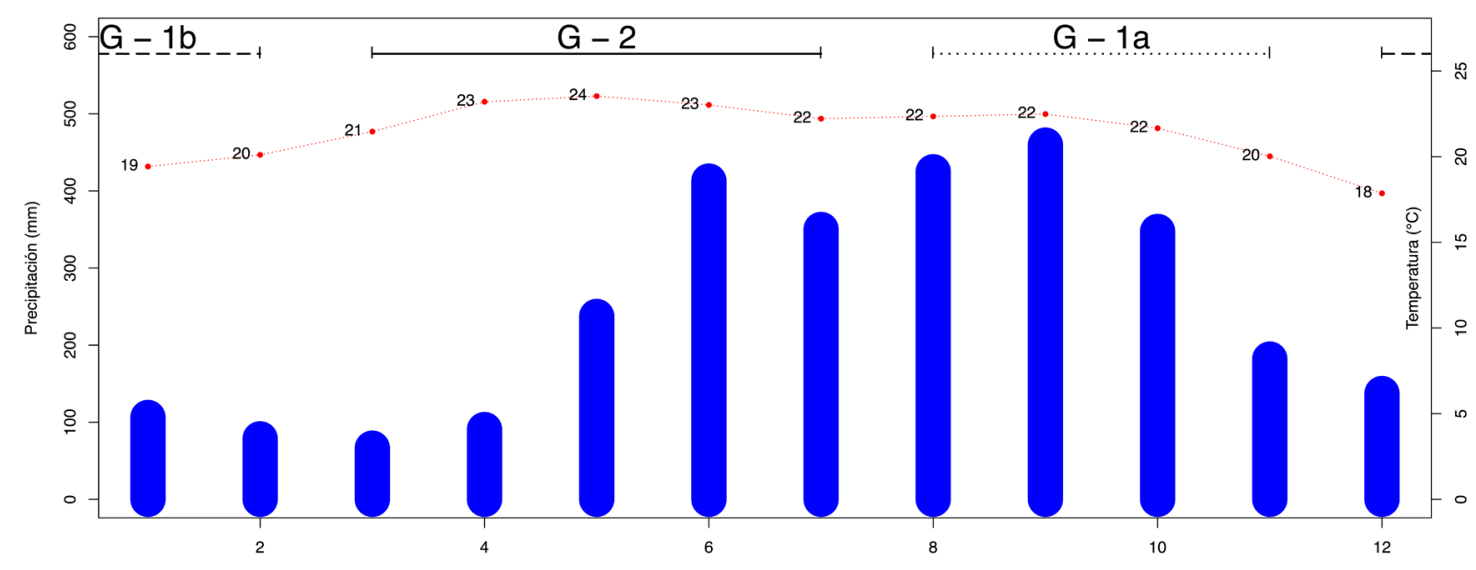

Meses

FIgURA 4. Grupos mensuales de floración de orquídeas en visitas a la zona de visitantes del PANACAM versus temperatura y precipitación (la línea roja punteada expresa la temperatura promedio mensual en grados centígrados, las barras azules muestran la precipitación promedio mensual, la línea negra punteada muestra la agrupación G-1a, la línea negra con espacios intercalados la agrupación G-1b y la línea negra sólida demarca la agrupación G-2 de floración).

PANACAM (Fig. 2), una cantidad que representa el $20 \%$ de las orquídeas de Honduras registradas por Nelson (2008, 2010), Archila (2013) y Vega et al. (2014), en un área que corresponde a 0.0001\% de la totalidad del PANACAM.

Aunque el objetivo de este estudio recae en la riqueza de las orquídeas de la zona de visitantes de PANACAM, es importante aportar las observaciones realizadas en cuanto a otras variables que nos ayuden a entender esta riqueza y su temporalidad.

Si se comparan las agrupaciones de meses contra la precipitación y temperatura, cada grupo parece estar relacionado con el clima (Fig. 4). G-1a presenta altas precipitaciones (181-424 $\mathrm{mm}$ ) y temperaturas promedio que van desde los 20 hasta $22^{\circ} \mathrm{C}$. G-1b presenta precipitaciones bajas $(78-137 \mathrm{~mm}) \mathrm{y}$ temperaturas promedio bajas $18-20^{\circ} \mathrm{C}$, las más bajas del año. Ambos subgrupos se podrían beneficiar del acumulado de humedad en el ambiente por los primeros meses de lluvia en el año. G-2 presenta precipitaciones bajas (66-237 $\mathrm{mm})$, exceptuando junio y julio, que son meses con precipitaciones altas (350-412 $\mathrm{mm})$, pero con temperaturas altas (21$23.5^{\circ} \mathrm{C}$ ), las más altas del año. Se podría emitir la hipótesis que la relación depende de la disponibilidad de agua, donde la precipitación representa la entrada de agua al sistema, y la temperatura, en forma de evaporación, la salida.

Estos resultados sugieren una relación entre el clima y la fenología de floración de las orquídeas, como menciona Dressler (1981), sin embargo esta sólo es la primera parte del proceso, donde el verdadero éxito de la especie se ve reflejado en la mayor posibilidad de germinación y consolidación de nuevos individuos. Plantas que dan flor en enero y plantas que dan flor en junio, suponemos tienen estrategias diferentes de preparación de fruto, dispersión de semillas y germinación, buscando las mejores condiciones ambientales para la germinación de su semilla y establecimiento de la planta (Dressler, 1981).

En base a que el área de estudio es pequeña y que de acuerdo a jackknife de primer orden se predice la presencia de más especies en el Parque, es necesario seguir este proceso de investigación de la historia natural de los organismos, dándole seguimiento al desarrollo de los frutos hasta el punto de la dispersión de su semilla y ver su relación con las variables climáticas. Para esto es de vital importancia la medición de disponibilidad de agua y otras variables que nos ayuden a entender de mejor forma la estrategia de estas plantas en el Parque. De igual forma, es necesario ampliar el área de investigación a la totalidad de PANACAM y procurar llegar a las partes más alta del parque donde se encuentra el bosque nublado donde estará la mayor diversidad de especies.

Los estudios a largo plazo, donde se puede abarcar mejor la riqueza de los grupos de plantas y animales, 
se perfilan de gran importancia para plantear planes de manejo efectivos de recursos naturales en las áreas protegidas hondureñas. Sin información de este tipo se puede dejar de lado las condiciones específicas de cada grupo y especie representados dentro de los límites que se desean proteger, lo que llevaría a una planificación a oscuras.

Agradecimientos. El autor quiere agradecer al personal del Proyecto Aldea Global por el apoyo y trato amable, a la Empresa Nacional de Energía Eléctrica, que a través de su departamento de meteorología, facilitó los datos climáticos de la estación meteorológica de Santa Elena, a J. L. Linares por la revisión de la determinación de gran parte de las especies, a J. E. Mérida y G. A. Cruz por la constante motivación, aporte de ideas y comentarios al manuscrito, a J. L. Murillo por la asistencia en cada viaje, a G. H. Wiese por ser inspiración sin saberlo y a dos revisores anónimos por los oportunos comentarios.

\section{LiTERATURA CITADA}

AFE-COHDEFOR. (1998). Plan de manejo, proyecto de áreas protegidas, Parque Nacional Cerro Azul Meámbar. Reporte técnico, Siguatepeque, Honduras.

AFE-COHDEFOR. (2000). Plan de manejo, proyecto de áreas protegidas, Parque Nacional Cerro Azul Meámbar. Reporte técnico, Siguatepeque, Honduras.

Archila, F. L. (2013). Lepanthes vilchezii nueva especie de Pleurothallidinae para Guatemala y Honduras. Revista Guatemalensis, 16(1), 12-21.

Borjas, G., Pérez, D. E., Aguilar, S., Thorn, S. L., Flores, S. \& Cruz G. A. (1997). Primer estudio sobre biodiversidad en el Parque Nacional Azul Meámbar. Reporte técnico, Proyecto Aldea Global.

C. V. Starr Virtual Herbarium. New York Botanical Garden. (2015). http://sciweb.nybg.org/science2/ VirtualHerbarium.asp, consultado el 15 de agosto 2015.

Chase, M. W., Cameron, K. M., Freudenstein, J. V., Pridgeon, A. M., Salazar, G. Van Den Berg C. \& Schuiteman, A. (2015). An updated classification of Orchidaceae. Botanical Journal of the Linnean Society, 177(2), 151-174.

Cruz, G. A. (1993). El decreto 87-87, ley de los bosques nublados, base legal para la conservación de los bosques nublados de Honduras. Serie Miscelánea de CONSEFORH 23. ESNACIFOR, Siguatepeque, Honduras.

Dressler, R. L. (1981). The orchids: natural history and classification. Harvard University Press, Cambridge, Massachusetts and London, England.

Dressler, R. L. (2005). How many orchid species? Selbyana, $26,155-158$.

Neotropical Herbarium Museum, The Field Museum (2015). http://fm1.fieldmuseum.org/vrrc/, consultado el 15 de agosto 2015.

Govaerts, R., Pfahl, J., Campacci, M. A., Holland-Baptista, D., Tigges, H., Shaw, J., Cribb, P., George, A., Kreuz, K. \& Wood, J. (2011). World checklist of Orchidaceae. The board of trustees of the Royal Botanical Gardens, Kew. Consultado diciembre 2014.

Hawkins, T. (1993). Informe del primer viaje al Parque Nacional de Azul Meámbar. Reporte técnico. 25-7/93, Serie Miscelanea de CONSEFORH, ESNACIFOR, Siguatepeque.

Hazlett, D. L. (1980). A botanical description of Cerro Azul Meámbar, Honduras. Brenesia 18, 201-206.

Holdridge, L. R. (1962). Mapa ecológico de Honduras. Reporte técnico, OEA, San José, Costa Rica.

Hágsater, E. \& Dumont V. eds. (1996). Status survey and conservation plan: orchids. UICN.

ICF (2012). Plan de manejo, proyecto de áreas protegidas, Parque Nacional Cerro Azul Meámbar. Reporte técnico, Siguatepeque, Honduras.

Nelson, C. H. (2008). Catálogo de plantas vasculares de Honduras, espermatófitas. Secretaria de Recursos Naturales y Ambiente / Guaymuras, Tegucigalpa.

Nelson, C. H. (2010). Adiciones y comentarios a la flora de Honduras. Ceiba 51(2), 70-88.

Nelson, C. H., \& Ortiz, J. C. (2007). La colección de orquídeas del herbario TEFH de Honduras. Ceiba, 48(1), 11-59.

Paradis, E., Claude, J. \& Strimmer, K. (2004). APE: analyses of phylogenetics and evolution in $\mathrm{R}$ language. Bioinformatics, 20, 289-290.

Peraza-Flores, L. N., Fernández-Concha, G. C. \& Romero-González G. A. (2011). Taxonomic notes in American Polystachya (Orchidaceae): The identity of P. foliosa (Hook.) Rchb.f. and the reestablishment of P. caracasana Rchb. f. The Journal of the Torrey Botanical Society, 138, 366-380.

QGIS Development Team. (2015). QGIS: Geographic information system. Open Source Geospatial Foundation.

R Core Team. 2013. R: A language and environment for statistical computing. R Foundation for Statistical Computing, Vienna, Austria.

Romero-González, G. A., Batista, J. \& L. de Bem Bianchetti. (2008). A synopsis of the genus Cyrtopodium (Catasetinae: Orchidaceae). Harvard Papers in Botany, 13, 189-206.

Tropicos.org, Missouri Botanical Garden. (2015), Agosto 15). http://www.tropicos.org, consultado 15 agosto del 2015. 
Vavrek, M. J. (2011). Fossil: palaeoecological and palaeogeographical analysis tools. Palaeontologia Electronica 14:1T. R package version 0.3.0.
Vega, H., Cetzal-Ix, W., Mó, E., Germer, D. \& Soler K. (2014). Nuevos registros de Orchidaceae para Honduras. Lankesteriana, 14, 115-121. 